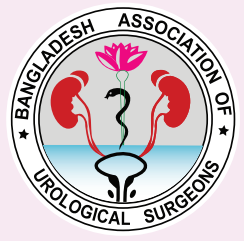

\title{
A Case Report on Bilateral Adrenal Histoplasmosis
}

\author{
Md. Sazzad Hossain ${ }^{1}$, Mohammad Zahid Hasan ${ }^{2}$, Mir Ehteshamul Haque ${ }^{3}$, Mostakim Maoya ${ }^{4}$
}

Received: 16 - 07 - 2020

Accepted: 30 - 09 - 2020

Conflicts of interest: None

Keywords: Histoplasma capsulatum, disseminated histoplasmosis, adrenal masses, hypoadrenalism.

\begin{abstract}
Disseminated histoplasmosis affecting the adrenal glands is a very rare infection and an uncommon cause of adrenal insufficiency. We present a case of bilateral adrenal histoplasmosis in a 77 years old immunosuppressed patient who presented with bilateral adrenal masses. The fine needle aspiration cytology of the adrenal mass was positive for Histoplasma capsulatum.
\end{abstract}

\section{Introduction:}

Histoplasmosis is a fungal disease caused by dimorphic fungus Histoplasma capsulatum $(\mathrm{H}$. Capsulatum). Histoplasmosis infection is endemic in certain part of the world including Asia (Benevides et al.,2007) ${ }^{1 \cdot}$. Histoplasmosis usually presents in 2 forms; pulmonary and extrapulmonary, which is also known as disseminated histoplasmosis (DH). Spores containing $\mathrm{H}$. capsulatum can be found in soils contaminated by droppings from birds and bats. Patients are infected via inhalation of these spores (Jombo et al., 2010. ${ }^{2}$ Disseminated histoplasmosis is uncommon but frequently ( $80 \%$ ) affects the adrenal glands (Goodwin et al.,1980) ${ }^{3}$. The incidence of hypoadrenalism amongst these group of patients is rare and was reported to be ranging between 7 to $20 \%$ (Subramanian et al., 2005; Rajesh et al., 2010). ${ }^{4-5}$. Hence, a high index of suspicion is prudent as the presentation may mimic other chronic infections or malignancy especially in the elderly or the immunosuppressed host (Jaiswal et al., 2011) ${ }^{6}$ We report a case of an elderly man with bilateral adrenal histoplasmosis and normal cortisol response who presented with vague symptoms.

\section{Case report:}

Mr. Bernard Gomes, 77 years old male an ex-nursing staff was living with pet cat for several years, Normotensive, Diabetic was admitted in Apollo Hospitals Dhaka (AHD) with severe generalized weakness for 2-3 months, about 10-12 kg weight loss in last 3 months, $\mathrm{H} / \mathrm{O}$ fever for 2 months with $\mathrm{H} / \mathrm{O}$ Bilateral PCNL \& right URS with LASER lithotripsy 3 month back. He was evaluated thoroughly \& relevant investigation done. ESR: $70 \mathrm{~mm}$ in $1^{\text {st }}$ hour, Urine: pus cells: Numerous, RBC 20-25/ HPF. Yeast cells: Nil / HPF. S. creatinine $2.3 \mathrm{mg} / \mathrm{dl}$. S. Cortisol: reduced. CT Urogram shows bilateral Adrenal mass, bilateral residual renal calculi with cyst, He underwent image guidance core biopsy of adrenal glands \& diagnosed bilateral Fungal Infection compatible with Histoplasmosis. MTB real time PCR- Mycobacterium tuberculosis (MTB) complex DNA: not detected, Nontuberculous mycobacteria (NTM): Detected. Patient was treated systemic antifungal, hydrocortisone \& other symptomatic medication by multidisciplinary team. Patient's condition was improved \& discharged with a stable condition. With given treatment patient shows poor response in first 2 months, thereafter he shows gradual improvement in

1 Registrar, Department of Urology, Evercare Hospital Dhaka

2. Senior Consultant, Department of Urology, Evercare Hospital Dhaka.

3. Senior Consultant, Department of Urology, Evercare Hospital Dhaka.

4. Registrar, Department of Urology, Evercare Hospital Dhaka.

Correspondences: Md. Sazzad Hossain, Registrar, Department of Urology, Evercare Hospital Dhaka-1229, Bangladesh, E-mail: sazzad.hossain@evercarebd.com 
terms of hunger, weight gain, general wellbeing. CT scan shows reducing size of Adrenal mass. We have advice to him to continue antifungal (Etaconazole) for one year \& Hydrocortisone to be continued and follow up three monthly.

\section{Discussion:}

Histoplasmosis is an infectious granulomatous disease caused by intracellular dimorphic fungus Histoplasma Capsulatum $^{4-5}$. Humans get infected by airborne spores of birds or bat's excreta ${ }^{4-5}$. The disease may be classified as Acute, Chronic, and Disseminated. Disseminated histoplasmosis is rare occurring mostly in acute rather than chronic forms, however, the frequency of dissemination in acute Histoplasma infections is 1:20006. Most patients who develop acute infection are immunocompromised. Adrenal involvement by histoplasmosis may occur during the active course of dissemination It could be either a part of disseminated form in immunosuppressed patients or may occur as a localized adrenal disease. Godwin et al. reported acute adrenal crisis in $7 \%$ of patients presenting with disseminated histoplasmosis ${ }^{12}$.

Adrenal histoplasmosis has a wide spectrum of clinical manifestations including chronic fatigue, weight loss, anorexia and low-grade fever, night sweats, and with the features of adrenal insufficiency. Primary adrenal insufficiency/Addison's disease occurs in $50-71 \%$ of adrenal histoplasmosis cases and is the commonest cause of death ${ }^{7}$. Physical examination may reveal hepatosplenomegaly or lymphadenopathies. Abdominal ultrasound revealed bilateral adrenal enlargement, the differential diagnosis included benign or malignant tumors, metastatic tumors, Lymphoma, adrenal hemorrhage, sarcoidosis and disseminated infections that include histoplasmosis, coccidioidomycosis and blastomycosis, $\mathrm{TB}^{6}$.

CT features of adrenal histoplasmosis include bilateral symmetric enlargement with preservation of normal contour, peripheral enhancement, and central hypodensity areas of necrosis and hemorrhage. These features are not specific for histoplasmosis and may occur in other disseminated infections like tuberculosis, cryptococcosis, and blastomycosis (paracoccidioidomycosis) and lymphoma. Although adrenal involvement can occur mostly unilateral involvement. Bilateral involvement is rare. Cytology or Histopathological examination of the adrenal mass after a fine needle aspiration or percutaneous biopsy which exhibits typical microscopic features of $H$. capsulatum would be the best method. H. capsulatum is an intracellular dimorphic fungus which is usually seen in the macrophages cytoplasm and exhibits narrow based budding (Fitzhugh et al., 2010[7]; Gopal et al., 2010 $)$. Our patient underwent adrenal biopsies. Histopathological examination from the adrenal biopsy consistent with histoplasmosis. Diagnosis involves performing enzyme immunoassay on urine, serum, cerebrospinal fluid. The gold standard for diagnosis of histoplasmosis is tissue culture ${ }^{9}$. Adrenal FNAC along with histopathology, culture, polymerase chain reaction, and urine antigen can confirm the diagnosis of histoplasmosis and can differentiate it from other conditions. The mortality in untreated disseminated histoplasmosis patients was as high as $80-100 \%$ but if treated with antifungal, this very high mortality rate is remarkably reduced to less than $25 \%$ (Subramanian et al., 2005 [10]). Majority of individuals who are asymptomatic with histoplasmosis recover spontaneously and do not require any specific treatment. Chronic and acute severe cases require treatment ${ }^{4}$. The recommended anti-fungal treatment is amphotericin B for critically ill hospitalized patients. This had to be replaced by itraconazole later because of nephrotoxicity. Itraconazole is well tolerated and has excellent central nervous system penetration. Ketoconazole may be used in milder presentations. Recurrence has been described as long as nine years after cessation of treatment, and therefore treatment duration of one to two years reduces the risk of relapse.

\section{Conclusion:}

Adrenal histoplasmosis does occur in immunocompromised patients and must be considered in the differential diagnosis of bilateral/unilateral adrenal masses presenting with adrenal insufficiency. A diagnosis of invasive fungal infection is critical and requires a high index of suspicion, especially in immunocompromised patients who have presented with nonspecific symptoms, clinical signs, and laboratory and radiological features resembling adrenal neoplasms. Clinical specimens must be sent for histopathology and fungal culture for a definite diagnosis and an appropriate management. Adrenal insufficiency has to be monitored and antifungal therapy should be maintained for at least 1 year to 2 years is recommended to reduce recurrence (Rana et al., $\left.2011^{14}\right)$. 


\section{References:}

1. Benevides CF, Duraes RO, Aquino B, Schiavon Lde L, Narciso-Schiavon JL, Buzzoleti Fda C.Bilateral adrenal histoplasmosis in an immunocompetent man. Rev Soc Brasil Med Trop. 2007;40:230-233. [PubMed] [Google Scholar]

2. Jombo GTA, Denen Akaa P, Banwat EB, Dauda M. A review of literature on unusual clinical presentations and potential challenges in diagnosis of histoplasmosis. J Clin Med Res. 2010;2:159-166.[Google Scholar]

3. Goodwin RA, Jr, Shapiro JL, Thurman GH, Thurman SS, Des Prez RM. Disseminated histoplasmosis: clinical and pathologic correlations. Medicine. 1980;59:1-33. [PubMed] [Google Scholar]

4. Subramanian S, Abraham OC, Rupali P, Zachariah A, Mathews MS, Mathai D. Disseminated histoplasmosis. J Assoc Physicians
India. 2005;53:185-189. [PubMed] [Google Scholar]

5. Rajesh KF, Warrier V, Gayathri R, Chellenton J, Geetha P, Thulaseedharan NK. A case of histoplasmosis. Calicut Med J. 2010; 8(2):e7. [Google Scholar]

6. Jaiswal S, Vij M, Chand G, Misra R, Pandey R. Diagnosis of adrenal histoplasmosis by fine needle aspiration cytology: an analysis based on five cases. Cytopathology: official journal of the British Society for Clinical Cytology. 2011;22:323328. [PubMed] [Google Scholar]

7. Fitzhugh VA, Maniar KP, Kim MK, Chen H, Schainker BA. Adrenal histoplasmosis. Diagn Cytopathol. 2010;38:188-189. [PubMed] [Google Scholar]

8. Gopal K, Singh S, Gupta R. Fine needle aspiration cytology in bilateral adrenal masses in a 58-yearold man. Acta Cytol. 2010;54:234-236. [PubMed] [Google Scholar]. 